\title{
Prostatic ductal adenocarcinoma: an unusual case of a rare prostate cancer
}

\author{
Rayan El Hassan,, John Corr, Rajiv Pillai \\ Department of Urology, Colchester Hospital University Foundation Trust, United Kingdom
}

Received: December 11, 2018

Accepted: April 4, 2019

Online Published: July 18, 2019

DOI: $10.5430 /$ jst.v9n2p28

URL: https://doi.org/10.5430/jst.v9n2p28

\begin{abstract}
A 65 year old gentleman was referred with symptoms of haematuria and haematospermia in association with an elevated prostate specific antigen (PSA). He was investigated with a flexible cystoscopy, Ultrasound scan and a computed tomography (CT) of his abdomen and pelvis. These failed to reveal any abnormality. Magnetic resonance imaging (MRI) revealed a Prostate Imaging Reporting and Data System PIRADS 2 lesion in the left peripheral gland and PIRADS 3 lesion on the right side posterolaterally at the level of mid gland of the prostate. He went on to have Transrectal ultrasound biopsies of his prostate (TRUS Bx) that excluded any pathology. On follow up visits his PSA continued to rise and he underwent Template biopsies of the prostate. The histological features had no evidence of any Prostatic intraepithelial carcinoma (PIN) or other malignancies. Flexible cystoscopy was repeated due to his persistent haematospermia. This showed prominent papillary lesions over his verumontanum and prostatic urethra. Biopsies from these areas revealed Ductal Adenocarcinoma of the Prostate (DACP). A subsequent staging MRI revealed unchanged appearance of the PIRADS2 nodule. There was however some low signal extending into the right seminal vesicle which is more pronounced than on the previous scan reported as PIRADS3. Subsequent mapping Template biopsies and Transurethral biopsies revealed a Gleason 4+4 DCAP. A staging CT and bone scan excluded any metastasis. He went on to receive an open radical prostatectomy and pelvic lymph node dissection as a curative treatment for his locally advanced disease.
\end{abstract}

Key Words: Ductal Adenocarcinoma of the Prostate, Prostate specific antigen, Flexible cystoscopy, Prostatic intraepithelial carcinoma, PIRADS, Gleason

\section{INTRODUCTION}

This case report illustrates a rare type of urological cancer with an unusual presentation. DACP is a rare type of cancer with no proven pattern of clinical behavior or progression. The case was unusual especially with the nonspecific symptoms that the patient has presented with. The disease was only diagnosed due to persistent symptoms and repeat investigations.

This case focuses on the unusual presentation and clinical behavior of DACP. The patient had haematuria, haematospermia and elevated PSA with a normal flexible cystoscopy. His initial investigations did not reveal any abnormality but a repeat flexible cystoscopy few months later established the papillary growth pattern within the periprostatic urethra. This is indicative of the aggressive nature of such type of cancer, highlighting the importance of repeat investigations in such cases.

The case also adopts on the management decision based on the histological findings and stage of the disease. The patient underwent an open radical prostatectomy and pelvic lymph nodes dissection following diagnosis of locally advanced pure DACP.

\footnotetext{
*Correspondence: Rayan El Hassan; Email: Rayan.ElHassan@ colchesterhospital.nhs.uk; Address: Department of Urology, Colchester Hospital University Foundation Trust, United Kingdom. 


\section{CASE PRESEntation}

A 65 year old patient was referred to our Urology department at Colchester General Hospital with visible haematuria and haematospermia and a presenting PSA of $8.6 \mathrm{ng} / \mathrm{ml}$. He was an Ex-smoker five years in cassation. He had a background history of diverticular disease and hypertension. The patient was first seen in the haematuria clinic. On examination, he had a 40cc benign prostate which was asymmetric with right lobe larger than left. A plain x-ray KUB, US of his urinary tract and flexible cystoscopy was normal. He underwent an MRI of his prostate and a Trans-rectal Ultrasound Guided biopsy of his prostate to investigate his elevated PSA. In the meantime he was admitted under the general surgical team with acute diverticulitis which was diagnosed on CT. The CT excluded any urological pathology. His MRI reported a $38 \mathrm{cc}$ prostate, a $1.7 \mathrm{~cm}$ rounded PIRADS 2 focus extending into left peripheral gland with some intermediate signal more keeping with hyper plastic nodule than tumour plus a small foci of restricted diffusion between right central and periphery gland posterolaterally could represent a small foci of organ confined tumour with PIRADS 3. It reported normal seminal vesicles (SV) and no lymphadenopathy or bony abnormalities. The histology report from the TRUS prostate biopsy revealed no evidence of malignancy. The patient was initially put on PSA surveillance. His follow up PSA was elevated at $14.31 \mathrm{ng} / \mathrm{ml}$ and evidently he received systematic template prostate biopsies, Histology had benign features with no evidence of PIN or any other malignancy. Subsequently his PSA dropped down to $11.6 \mathrm{ng} / \mathrm{ml}$ but the haematospermia persisted. A repeat flexible cystoscopy revealed prominent papillary lesions around the verumontanum within the prostatic urethra. The papillary lesions were biopsied at the same time. Histology revealed Ductal Carcinoma of the Prostate. His case was then discussed in our Multidisciplinary Team Meeting (MDT). The outcome of the meeting was to offer him a staging MRI scan and a formal transurethral (TUR) biopsy. His MRI reported a $54 \mathrm{cc}$ prostate and an unchanged hyperplastic PIRADS2 nodule over left peripheral gland. There was however a low signal extending into the right seminal vesicle which was more pronounced than on previous scan with PIRADS3. The central gland was non suspicious. The seminal vesicles appeared otherwise normal and had no lymphoadenopathy or any bony abnormalities. He went on to have repeat systematic Template biopsies as well as TUR biopsies of the prostatic urethra under general anaesthesia. The resection biopsies were done at the veumontanum and twenty template biopsies done including the right SV. The histology report showed "Ductal Adenocarcinoma equivalent to Gleason of " $4+4=8$ ", present in $7 / 54$ cores. The left medial anterior apex

Published by Sciedu Press
$(60 \%)$ and left lateral prostate $(50 \%)$ zones were positive. There was no evidence of perineurial invasion. The targeted Right SV biopsy included fragments of Ductal Adenocarcinoma." Following these findings, further staging including a staging CT of chest, abdomen and pelvis $\&$ a bone scan revealed no metastatic disease (T3bNoMo). His case was discussed in the specialist network MDT and it was decided to offer all active treatment options with a curative intent including surgery, external beam radiotherapy and HDR (high dose rate brachytherapy) boost. The patient opted for radical surgery after appropriate counselling including the possibility of adjuvant treatment following radical surgery. The patient underwent an uneventful Open Radical Prostatectomy and Pelvic lymph nodes dissection. His prostatectomy specimen showed approximately $35 \%$ total volume of left side of the prostate being occupied with invasive Gleason $4+4$ ductal carcinoma of the prostate, with no perineurial or vascular invasion readily identified (pT2a pN0 pR0). There was no extension of the disease beyond the prostate, the SVs and no metastasis or any lymph nodes involvement. His surgery and recovery were uneventful. He is under close follow up after surgery. He had mild stress urinary incontinence postoperatively which resolved completely on subsequent follow ups. His PSA has been undetectable and had a clear 6 monthly CT scan. The patient was free of recurrence or metastatic disease at 12 months follow up and hasn't received any adjuvant or salvage therapy. He is to receive a further CT scan at two and five years. The patient is also under regular cystoscopic surveillance. The close follow-up and monitoring is very important so that any early recurrent or metastatic disease is detected.

\section{DISCUSSION}

Whilst prostate cancer is a common cancer in men, multiple variants are present and some of which are atypical. Some of these atypical variants have been previously reported to present with low PSA. ${ }^{[1]}$ Ductal Adenocarcinoma of the Prostate was first reported in in 1967 by Melicow and Patcher presumed to rise from mullarian remnants and described as an endometrioid cancer. ${ }^{[2]}$ It was later in 2007 that Orihuela and Green have confirmed its prostatic stem. DACP is considered a rare yet aggressive form of prostate carcinoma. It's mainly found on incidental biopsy or histological outcomes from prostatectomies. In a large series, patients with ductal variant presented more with low PSA levels and more advance stage of the disease ${ }^{[3]}$ With its site of growth, DCAP manifests with haematuria and symptoms of obstruction difficult to distinguish from BPH. It can be detected on cystoscopy showing papillary changes over the periprostatic urethra and near the verumontanum utricle. It 
is mainly found in the periprostatic urethra and suggestive of typical growth into the periurethral ducts and stroma. The site of growth is the only reported feature of this type of cancer. It can be present as pure disease or mixed; typically with Acinar adenocarcinoma. ${ }^{[4]}$

Patients with high PSA levels above the specific age reference or visible haematuria require an urgent referral as per NICE Guidelines. The patient had also presented with haematospermia. A single episode of haematospermia is likely to be benign or self-limiting. ${ }^{[5]}$ On the other hand, high risk patients with repeatedly elevated PSA and persistent symptoms should have an urgent referral and further investigations (NICE and BAUS). A thorough examination including DRE, endoscopic examinations, TRUS Biopsy and imaging including CT and MRI should be carried out to exclude any malignancies. ${ }^{[6]}$ Though some has criticized the diagnostic profit of flexible cystoscopy and ultrasound scan as an initial investigation for haematosperimia. ${ }^{[7]}$ In this scenario, the patient received a cystoscopy and ultrasound scan as part of his haematuria investigations. As for the CT; that was done whilst investigating his acute diverticulitis. The patient went on to have his PSA monitored as part of PSA surveillance. Template biopsies were carried on based on previous negative investigations including non conclusive imaging results and the PSA trend. Flexible cystoscopy was repeated due to persistent haematospermia Biopsies were taken then due the suspicious papillary lesions around the verumonatum within prostatic urethra. DCAP is commonly picked up incidentally from prostatectomy and biopsy specimens. ${ }^{[8]}$ It mainly presents at the prostatic urethra; that being the only specific feature with its histological variant growth. ${ }^{[9]}$ After the diagnostic findings of DACP, a transurethral resection biopsies, template biopsies and MRI scan were done followed by a CT of the chest, abdomen and pelvis. The investigations were done to find out if the disease was pure or mixed DACP and assess the extent of the disease or any metastatic involvement. After the diagnostic findings of DACP, a transurethral resection biopsies, Template biopsies and MRI scan were done followed by a CT of the chest, abdomen and pelvis. The investigations were done for the differential between pure or mixed DACP and assess the extent of the disease or any metastasis.

The patient presented with mixed urological symptoms. He had symptoms of visible haematuria as well as haematospermia. His PSA was also elevated with a presenting PSA of $8.6 \mathrm{ng} / \mathrm{ml}$. The mixed clinical picture posed a diagnostic dilemma initially. Hence he underwent a variety of preliminary investigations to rule out both upper and lower urinary tract pathologies. These investigations ruled out renal cancer, stones, malformations and any other upper tract pathologies.
The primary flexible cystoscopy was normal which ruled out any urethral and bladder pathologies or other lower urinary tract abnormalities. Repeated investigations which proved normal, posed a diagnostic challenge. Prostate disease was suspected with the persistent haematospermia and elevated PSA. The standard workup for prostate cancer as per EAU guidelines includes MRI and prostate biopsies via transrectal or transperineal route. Despite the initial negative investigations, the patient underwent repeat investigations due to persistent symptoms. This enabled the diagnosis of a DCAP through flexible cystoscopy by detecting changes within the prostatic urethra. There is no clear management strategy for DCAP yet due to uncommon nature of DCAP and the poor understanding of its natural history. It has been reported that the risk of positive margin in DCAP is higher due to its extension into the urethral submucosa. ${ }^{[10]}$ The patient was diagnosed with locally advanced ductal adenocarcinoma of the prostate with involvement of the right seminal vesicle. There was no bony involvement and no metastasis on initial work up. After a multidisciplinary team meeting discussion, a close consultation was undertaken with the patient in the outpatient department. The patient was given the option of having radical treatment with a curative intent including surgery, external beam radiotherapy or high dose rate brachytherapy as the primary treatment option. He was counselled that he's likely to need a multimodality treatment considering the aggressive nature of the tumour.

Our patient had an unusual presentation of DCAP. He had unique features and variant clinical behaviour. His persistent haematospermia lead to repeated investigations which led to the diagnosis eventually. He had an organ confined disease with no extra capsular involvement and no metastasis. The behavior of this disease has been difficult to confirm due to its rarity. There has been evidence to suggest it might behave more aggressively than other variants like Acinar adenocarcinoma. A large study in 2010 did show that patients with DACP more commonly awarded distant and poorly differentiated disease. It also resulted in more extra capsular presentation yet not particularly different in the rates of lymph nodes involvement. ${ }^{[2]}$

Tu SM and Pisters LL reported that patients with no mixed ductal cancers survived longer after radical surgery with the disease resulting in an increased likelihood for local recurrence. The clinical outcome of pure variants may improve with prostatectomy in particular. Unfortunately in some cases, metastasis and extra-capsular extension with positive margins can develop with the disease being diagnosed at late stages, with liver and lung being a common site of spread. ${ }^{[11]}$ Such cases have been reported previously. Systemic chemotherapy proceeded with secondary hormonal 
treatment was suggested in these cases. ${ }^{[12]}$ DCAP is a rare and aggressive disease with an atypical presentation and clinical behavior. Due to its rarity and common late presentation, there has been no clear algorithm or guidelines for diagnosing and managing these patients. Thorough and repeated investigations, especially in the presence of recurrent symptoms is the key to diagnosis of this rare condition. Multidisciplinary

\section{REFERENCES}

[1] Leibovici D, Spiess PE, Agarwal PK. Prostate cancer progression in the presence of undetectable or low serum prostate-specific antigen level. Cancer: Interdisciplinary International Journal of the American Cancer Society. 2007 Jan; 109(2): 198-204. PMid:17171704. https://doi.org/10.1002/cncr. 22372

[2] Melicow MM, Patcher MR. Endometrial carcinoma of prostatic utricle (uterus masculinus). Cancer: Interdisciplinary International Journal of the American Cancer Society. 1967; 20: 1715-1722.

[3] Todd MM, Christopher JW, Funda VL. Ductal adenocarcinoma of the prostate: increased mortality risk and decreased PSA secretion. The Journal of Urology. 2010; 184(6): 2303-2307. PMid:20952027. https://doi.org/10.1016/j.juro.2010.08.017

[4] Bock BJ, Bostwick DG. Does prostatic ductal adenocarcinoma exist? American Journal of Surgical Pathology. 1999 Jul; 23(7): 781-5. PMid:10403300. https ://doi .org/10.1097/00000478-19990 7000-00005

[5] Fuse H, Komiya A, Nozaki T, et al. Hematospermia: etiology, diagnosis, and treatment. Reproductive Medicine and Biology. 2011 Oct; 10(3): 153-9. PMid:29699089. https://doi.org/10.1007/ s12522-011-0087-4

[6] Magoha GA, Magoha OB. Aetiology, diagnosis and management of haemospermia: a review. East African Medical Journal. 2008; 84(12). https://doi.org/10.4314/eamj.v84i12.9596 involvement and thorough consultation with the patient is required, highlighting the prognosis, and unpredictable nature of the tumour. The primary treatment approach has to be radical and aggressive with a curative intent but very often a multimodality management is required.

\section{Conflicts of Interest Disclosure}

The author declares no conflict of interest
[7] Ng YH, Seeley JP, Smith G. Haematospermia as a presenting symptom: outcomes of investigation in 300 men. The Surgeon: Journal of the Royal Colleges of Surgeons of Edinburgh and Ireland. 2013; 11(1): 35-38. PMid:22682581. https://doi.org/10.1016/j.su rge. 2012.04.004

[8] Tantan L, Yingmei W, Ru Z. The update of prostatic ductal adenocarcinoma. Chinese Journal of Cancer Research. 2016; 28(1): 50-57.

[9] Seipel AH, Wiklund F, Wiklund NP. Histopathological features of ductal adenocarcinoma of the prostate in 1,051 radical prostatectomy specimens. Virtuous Archiv:The European Journal of Pathology. 2013 Apr; 462(4): 429-36. PMid:23443941. https : //doi .org/10 .1007/s00428-013-1385-5

[10] Perrapato SD, Shah PC, Huben RP. Locally recurrent endometrioid adenocarcinoma of the prostate after radical prostatectomy. The Journal of Urology. 1991 Sep; 145(2): 373-375. https : //doi . org/10 $.1016 / \mathrm{S} 0022-5347$ (17) 38345-3

[11] Tu SM, Lopez A. Leibovici D Ductal adenocarcinoma of the prostate: clinical features and implications after local therapy. Cancer: Interdisciplinary International Journal of the American Cancer Society 2009 Jul; 115(13): 2872-80. PMid:19402048. https://doi .org/ $10.1002 /$ cncr. 24326

[12] Kumar A, Mukherjee SD. Metastatic ductal carcinoma of the prostate: a rare variant responding to a common treatment. Canadian Urological Association journal 2010; 4(2): 50-4. https://doi.org/10.5 $489 /$ cuaj. 830 